\title{
Adaptive robust control of unmanned helicopter angular rate
}

\author{
Xingwen Zhang ${ }^{1, \text { a }}$, Ming Chen ${ }^{1, \mathrm{~b}}$, PeiZhi Liu' ${ }^{2}$, Jinhua Wang ${ }^{2}$, Yi Qian ${ }^{2}$, \\ Xianxiang Chen ${ }^{2, c}$ \\ ${ }^{1}$ School of Aeronautic Science and Engineering, Beijing University of Aeronautics and Astronautics, \\ Beijing 100191, China \\ ${ }^{2}$ China ordnance industry computer application technology research institute, Beijing 100089, China \\ azhangxingwen@hrbeu.edu.cn, ${ }^{\mathrm{b}}$ chenming@buaa.edu.cn, ${ }^{\mathrm{c}}$ chenxx_710@126.com
}

Keywords: extended state observer, unmanned helicopter, sliding-mode control, uncertain system.

\begin{abstract}
Put forward a compound control strategy, which is designed with extended state observer and sliding mode variable structure control. The angular rate control of unmanned helicopter is validated through a Hardware-in-the-loop simulation, the simulation results show that the proposed control strategy can not only make the angular rate control of helicopter stable under the flight condition of turbulent, but also can ensure the angular rate accurate tracking according to different helicopter model without changing model parameters. The results indicate that the controller has strong robustness and adaptive ability.
\end{abstract}

\section{Introduction}

Unmanned helicopter system have seen unprecedented levels of growth during the last two decades. It's widely used in military, civilian and public applications. The autonomous flight control as one of the major areas of helicopter research have attracted much attention.

The helicopter system has complex aerodynamics characteristics, is a strong coupling multivariable nonlinear time-varying system, its model is too complex to be established. It brings the challenge to autonomous flight controller design. in view of the unmanned helicopter controller design problem, the domestic and foreign scholars have conducted a lot of research work.

Many methods have been applied to the unmanned helicopter controller design such as: PID control [1], the linear quadratic (LQ) optimal control [2], H-infinity robust control [3], and model predictive control (MPC) [4], the dynamic inversion (NDI) [5], Back-stepping (BS) [6] et al. Where, the PID algorithm is easy to implement, it's parameters can be setted in the flight, but it's robustness is relatively poor as LQ. H-infinity control is a kind of robust algorithm has strong robustness. Nonlinear dynamic inversion and back-stepping is able to control nonlinear model,but its implementation needs accurate model of helicopter which is difficult to establish.

We put forward a compound control strategy in this paper, which is designed with extended state observer and sliding mode variable structure control. The angular rate control of unmanned helicopter is validated through a Hardware-in-the-loop simulation, the simulation results show that the proposed control strategy can not only make the angular rate control of helicopter stable under the flight condition of turbulent, but also can ensure the angular rate accurate tracking according to different helicopter model without changing model parameters. The results indicate that the controller has strong robustness and adaptive ability.

\section{Controller Design}

In order to overcome the problem that the aerodynamic characteristics of unmanned helicopter is too complex to establish an accurate model, this paper put forward a kind of compound control strategy, which is designed with extended state observer and sliding mode variable structure control. Where, the sliding mode variable structure control can guarantee the stability of the modeled helicopter dynamic and robustness of system with parameter perturbation, extended state observer is 
used to observe and compensate the disturbance caused by un-modeled dynamics. The control structure diagram is showed in figure 1.

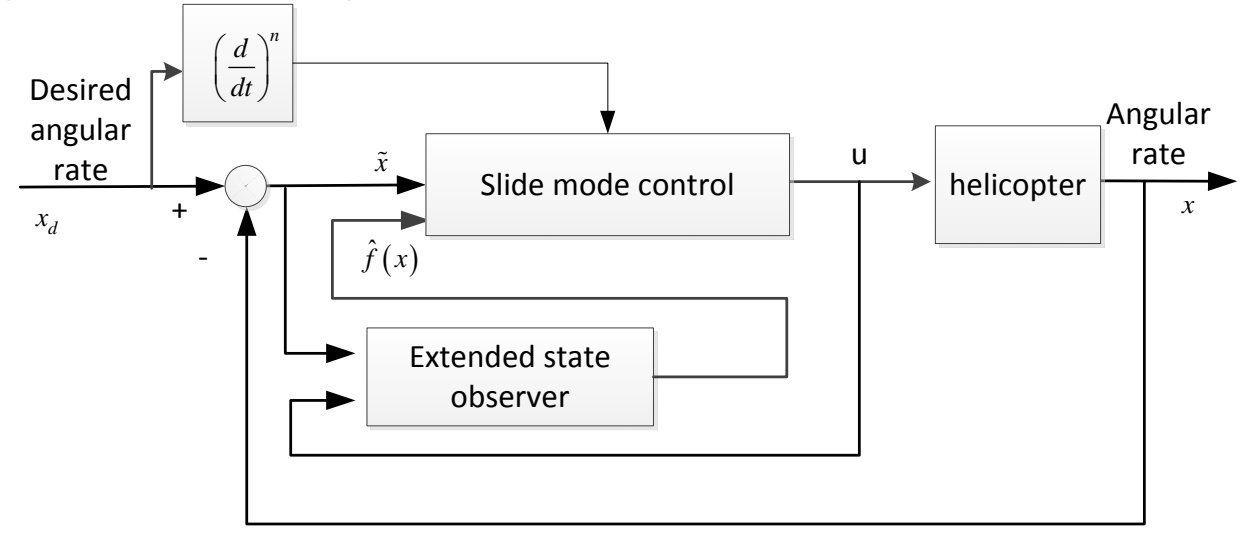

Fig 1 Control structure diagram

\subsection{Sliding mode control.}

Helicopter pitching and roll angular rate affected by lateral and longitudinal wave is second order nonlinear system, and yaw angular rate system is first order nonlinear model, the model of helicopter angular rate could be written as the following form:

$$
x^{(n)}=f(\boldsymbol{x})+b(\boldsymbol{x}) U
$$

Denote $\tilde{\boldsymbol{x}}=\boldsymbol{x}-\boldsymbol{x}_{\boldsymbol{d}}=\left[\begin{array}{cccc}\tilde{x} & \dot{\tilde{x}} & \cdots & \tilde{x}^{(n-1)}\end{array}\right]^{T}$ as tracking error of the state $\mathrm{x}$, denote the sliding mode surface $\mathrm{S}(\mathrm{t})$ :

$$
s=\left(\frac{d}{d t}+\lambda\right)^{n-1} \tilde{x}
$$

$\lambda$ is positive constant in the last written. The sliding condition is showed as below:

$\frac{1}{2} \frac{d}{d t} s^{2} \leq-\eta|s|$

For second order system:

$$
\dot{s}=\ddot{x}-\ddot{x}_{d}+\lambda \dot{\tilde{x}}=f(x)+b U-\ddot{x}_{d}+\lambda \dot{\tilde{x}}
$$

The estimated $\hat{U}$ is [7]:

$$
\hat{U}=-\hat{f}(x)+\ddot{x}_{d}-\lambda \dot{\tilde{x}}
$$

where, superscript" $\sim$ "denote the error between current state signal and measured state signal, subscript "d"denote the desired signal. Determine a switched control law to guarantee the sliding condition:

$$
U=\hat{b}^{-1}(\hat{U}-k \operatorname{sgn}(s))
$$

Where:

$$
\operatorname{sgn}(s)=\left\{\begin{array}{c}
1, s>0 \\
-1, s<0
\end{array}\right.
$$

Thus:

$$
\dot{s}=\left(f-b \hat{b}^{-1} \hat{f}\right)+\left(1-b \hat{b}^{-1}\right)\left(-\ddot{x}_{d}+\lambda \dot{\tilde{x}}\right)-b \hat{b}^{-1} k \operatorname{sgn}(s)
$$

Denote parameter perturbation range as:

$$
|f-\hat{f}| \leq F, B^{-1} \leq \frac{\hat{b}}{b} \leq B, B>1
$$

$\mathrm{k}$ should satisfy the following conditions:

$$
k \geq B F+\eta B+(B-1)\left(\hat{f}-\ddot{x}_{d}+\lambda \dot{\tilde{x}}\right)
$$


In order to reduce the control flutter caused by sign function, take saturation function $\operatorname{sat}(s / \Phi)$ instead of $\operatorname{sgn}(s)$ 。

$$
\operatorname{sat}(s / \Phi)= \begin{cases}s / \Phi, & |s / \Phi| \leq 1 \\ \operatorname{sgn}(s / \Phi), & |s / \Phi|>1\end{cases}
$$

\subsection{The Extended State Observer.}

The essence of the extended state observer is borrowed from the ideas of the state observer, extend the disturbence as a new state which coulde be observe. 14-15]。For the second order system, the extended state observer is [8]:

$$
\begin{aligned}
& \left\{\begin{array}{l}
e=z_{1}-y \\
\dot{z}_{1}=z_{2}-\beta_{01} e \\
\dot{z}_{2}=z_{3}-\beta_{02} \operatorname{fal}(e, 0.5, \delta)+b u \\
\dot{z}_{3}=-\beta_{03} \operatorname{fal}(e, 0.25, \delta)
\end{array}\right. \\
& \text { Where, } \operatorname{fal}(e, \varepsilon, \delta)= \begin{cases}\frac{e}{\delta^{\varepsilon-1}}, & |e| \leq \delta \\
|e|^{\varepsilon} \operatorname{sign}(e), & |e|>\delta\end{cases}
\end{aligned}
$$

The parameter is selected as below:

$\beta_{01}=3 \omega, \beta_{02}=3 \omega^{2}, \beta_{03}=\omega^{3}$

The output $z_{3}$ is estimation of the disturbence。

In the same way,for the first order system, the extended state observer is:

$$
\left\{\begin{array}{l}
e=z_{1}-y \\
\dot{z}_{1}=z_{2}-\beta_{01} e+b u \\
\dot{z}_{2}=-\beta_{02} \text { fal }(e, 0.5, \delta)
\end{array}\right.
$$

The parameter could be:

$\beta_{01}=2 \omega, \beta_{02}=\omega^{2}$

\section{Simulation Results.}

This paper uses the hardware-in-the-loop simulation system to verify this control algorithm,In order to prove the adaptability of the control strategy, two different helicopter model is chosen as control object in X-plane which is Mosquito XE(Fig.2 left)and Raptor30(Fig.2 right).
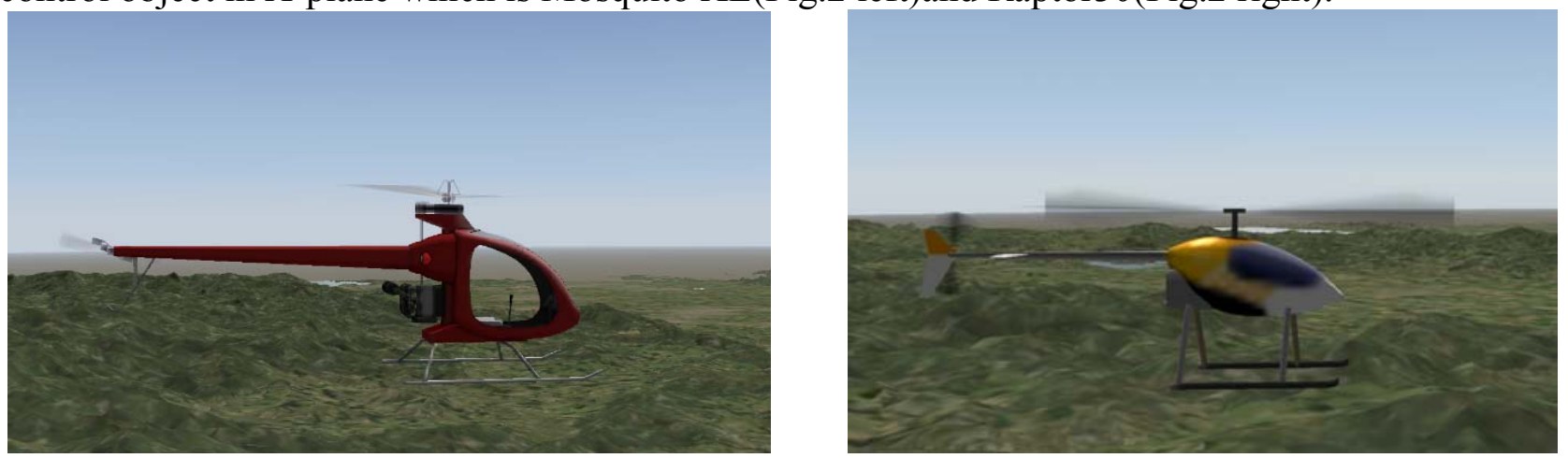

Fig. 2 Control Simulation of Helicopter in X-plane

Select controller parameters as: $\lambda=2, \eta=0.5, B=1.2, \omega=5, \hat{b}_{1}=58, \hat{b}_{2}=70, \hat{b}_{3}=100, \Phi=0.2$, the simulation result is shown as below: 

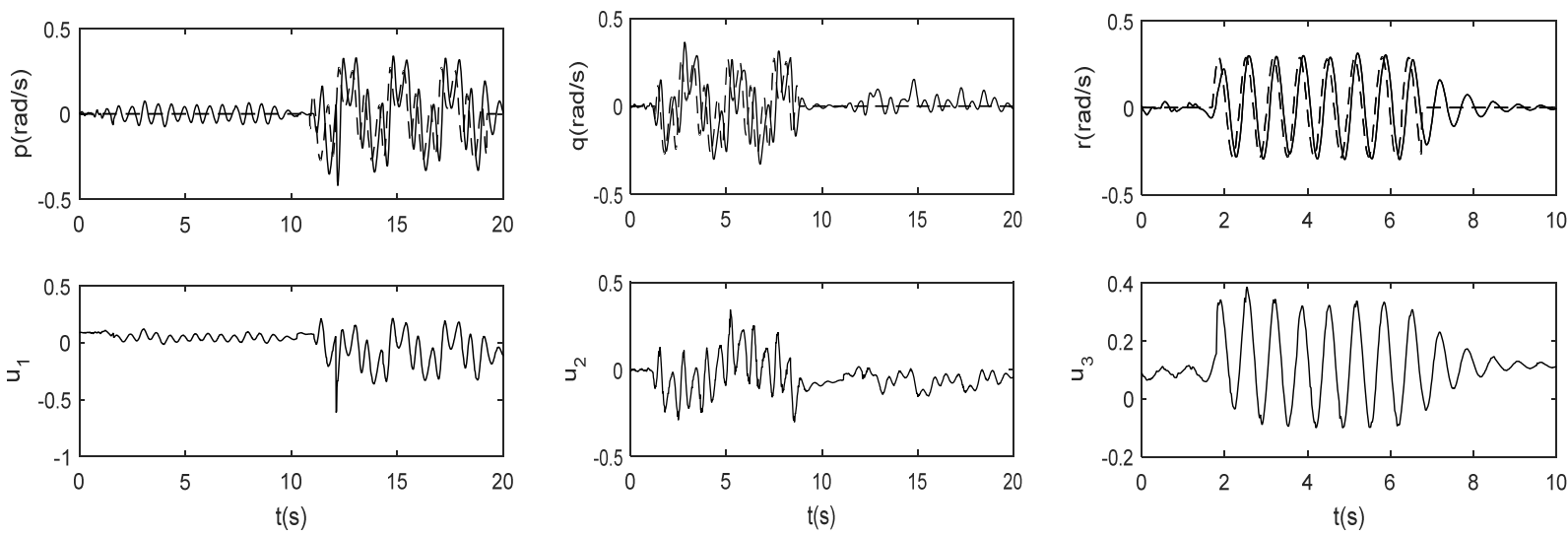

Fig. 3 Control result of Mosquito XE
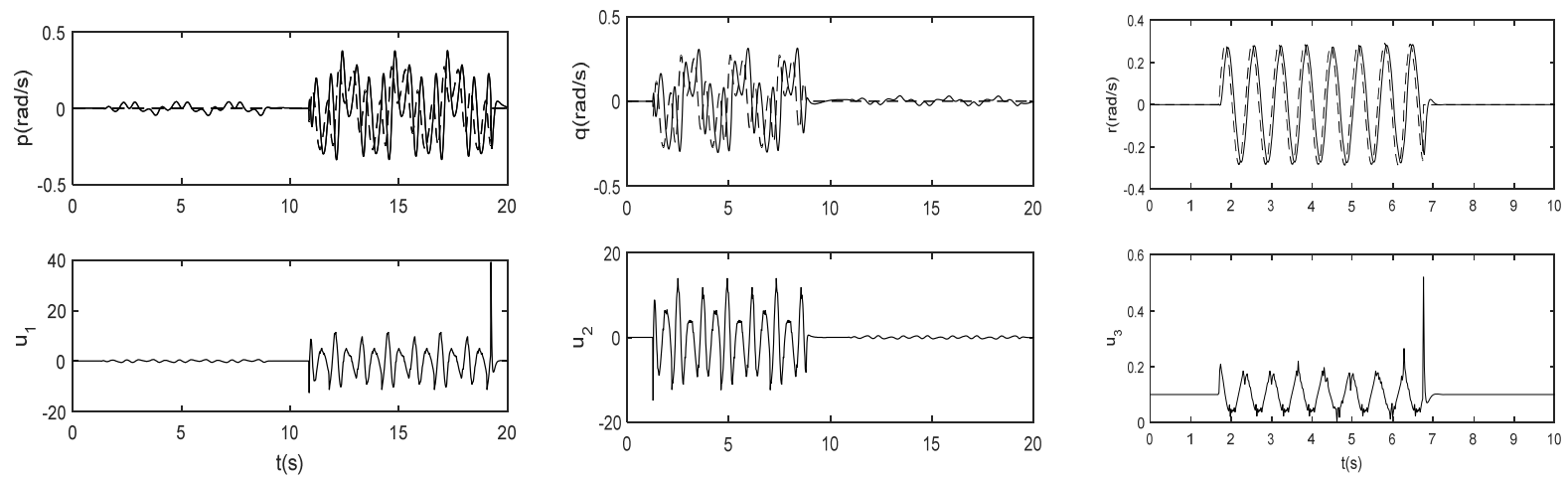

Fig. 4 Control result of Raptor30

The figure 3 showed angular control resul of Mosquito XE without wind. The picture above are agular rate tracking, where the dotted line is desired angular rate, and the solide line is current angular rate. The picture below are control signals respectively in three axis. Figure 5 is the angle rate control of raptor30 in turbulent condition. The simulation results indicate that the controller has strong robustness and adaptive ability.

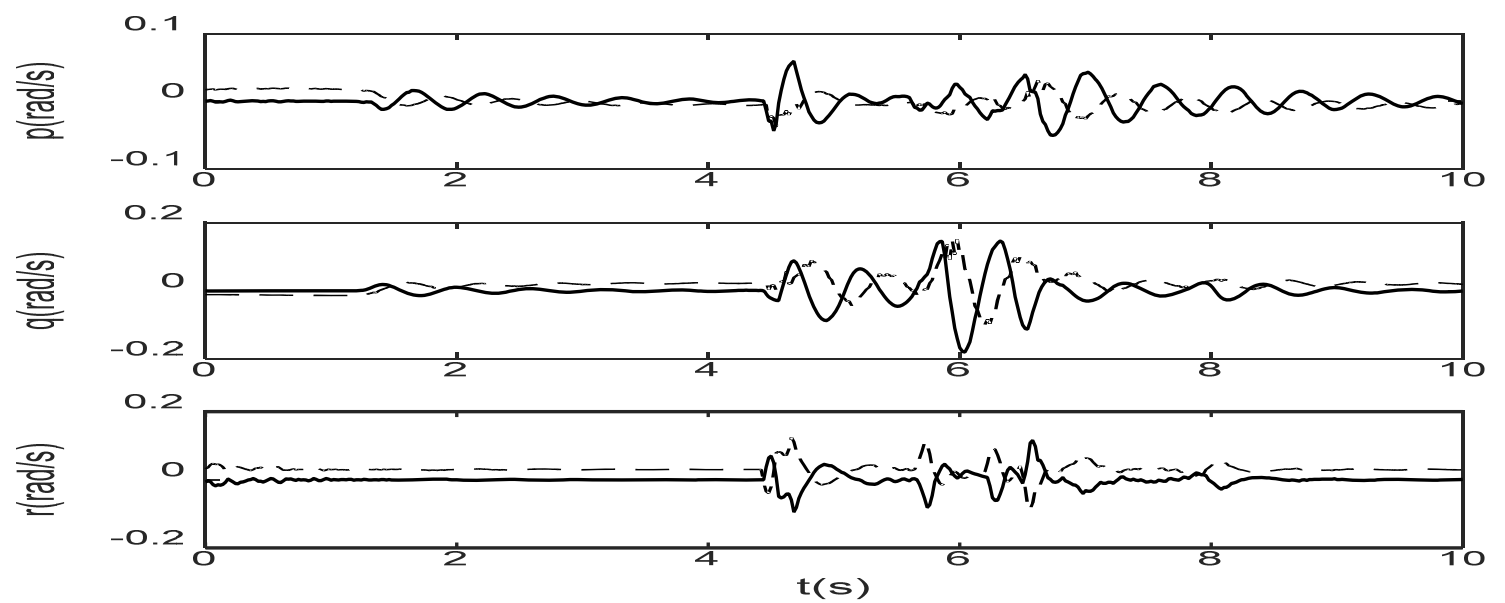

Fig. 5 Angle Rate Control of Raptor30 in Turbulent Condition

\section{Summary}

In view of the unmanned helicopter dynamic characteristic is complex, put forward an unmanned helicopter angular rate control strategy based on extended state observer and sliding mode control. Hardware-in-the-loop simulation is taken to verify the performance of the controller, the simulation results indicate that the controller has strong robustness and adaptive ability. 


\section{References}

[1]. Godbolt, B., Lynch, A.F. Control-oriented physical input modelling for a helicopter UAV. Intel. Robot. Syst. Vol 73 (2014) p: 209-217.

[2]. Lee, D.J., Bang, H.: Model-free LQ control for unmanned helicopters using reinforcement learning, 11th International Conference on Control. Auto. Syst. 6, 2011,p:117-120

[3]. Chen X., Liu Y.et al.: Modeling and attitude control of the miniature unmanned helicopter Measurement\& Control Technology, Vol 33(2014), No.3, p: 86-89.

[4]. Samal, M.K., Garratt, M., Pota, H., Teimoori, H.: Model predictive flight controller for longitudinal and lateral cyclic control of an unmanned helicopter // 2nd Australian Control Conference, November, 2012, p:. 386-39

[5]. Simplicio, P., Pavel, M., et al. An acceleration measurements-based approach for helicopter nonlinear flight control using Incremental Nonlinear Dynamic Inversion. Control Eng. Pract. 2013.21(8) p:1065-1077.

[6]. Raptis, I.A., Valavanis, K.P., et al: A Novel Nonlinear Back-stepping Controller Design for Helicopters Using the Rotation Matrix. IEEE Trans. Control Syst. Technol.2011, 19(2): 465-473.

[7]. Jean-Jacques E. Slotine Weiping Li. Applied Nonlinear Control. Pearson Education, Inc. Prentice Hall International Inc. 1991

[8]. Han Jingqing. Active Disturebance Rejection Control Technique-the technique for estimation and compensationg the uncertainties.National Defense Industry Press, 2008. 